\title{
Maternal and perinatal Health Research Collaboration, India (MaatHRI): establishing a hospital-based international collaborative research platform
}

Manisha Nair ${ }^{1}$, Babul Bezbaruah ${ }^{2}$, Amrit Bora ${ }^{3}$, Krishnaram Bora ${ }^{4}$, Shakuntala Chhabra ${ }^{5}$, Saswati Choudhury ${ }^{6}$, Arup Choudhury ${ }^{7}$, Dipika Deka ${ }^{8}$, Gitanjali Deka ${ }^{9}$, Vijay Anand Ismavel $^{10}$, Swapna Kakoty ${ }^{11}$, Roshine Koshy ${ }^{10}$, Pramod Kumar ${ }^{5}$, Pranabika Mahanta ${ }^{12}$, Robin Medhi ${ }^{11}$, Pranoy $\mathrm{Nath}^{2}$, Anjali Rani ${ }^{13}$, Indrani Roy ${ }^{14}$, Usha Sarma ${ }^{9}$, Carolin Solomi ${ }^{10}$, Ratna Talukdar ${ }^{6}$, Farzana Zahir ${ }^{15}$, Michael Hill ${ }^{1}$, Nimmi Kansal ${ }^{16}$, Reena Nakra $^{16}$, Colin Baigent ${ }^{1}$, Marian Knight ${ }^{17}$, and Jennifer Kurinczuk ${ }^{1}$

${ }^{1}$ University of Oxford

${ }^{2}$ Silchar Medical College and Hospital

${ }^{3}$ Mahendra Mohan Choudhury Hospital

${ }^{4}$ Nagaon Bhogeswari Phukanani Civil Hospital

${ }^{5}$ Mahatma Gandhi Institute of Medical Sciences

${ }^{6}$ Gauhati Medical College and Hospital

${ }^{7}$ Dhubri Civil Hospital

${ }^{8}$ Srimanta Sankaradeva University of Health Sciences

${ }^{9}$ Tezpur Medical College

${ }^{10}$ Makunda Christian Leprosy and General Hospital

${ }^{11}$ Fakhruddin Ali Ahmed Medical College and Hospital

${ }^{12}$ Jorhat Medical College and Hospital

${ }^{13}$ Banaras Hindu University Institute of Medical Sciences

${ }^{14}$ Nazareth Hospital

${ }^{15}$ Assam Medical College and Hospital

${ }^{16}$ Dr Lal PathLabs

${ }^{17}$ National Perinatal Epidemiology Unit

May 12, 2020

\begin{abstract}
Objective: To describe the processes used to establish and standardise a UK-India collaborative platform for maternal and perinatal health research (MaatHRI). Design: Hospital-based research platform. Setting: India Population: Pregnant women Methods: MaatHRI is adapted from the UK Obstetric Surveillance System (UKOSS) and built on a pilot model (IndOSSAssam), which has been extensively standardised using the following methods: (i) establishing a network of hospitals; (ii) setting up a secure system for data collection, storage and transfer; (iii) developing a standardised laboratory infrastructure; and (iv) developing and implementing regulatory systems. Results: MaatHRI was established in September 2018. Fourteen hospitals participate across four states in India - Assam, Meghalaya, Uttar Pradesh and Maharashtra. The Indian research team includes 20 nurses, a project manager, 16 obstetricians, two pathologists, a public health specialist, a general physician and a paediatrician. The UK partners include clinicians and epidemiologists from the University of Oxford. MaatHRI has
\end{abstract}


advanced standardisation of data and laboratory parameters, monitoring data and participant safety, and secure transfer of data. An integral component of MaatHRI is bi-directional capacity building. It is overseen by a steering committee and a data safety and monitoring board which ensure equitable partnerships between the collaborating institutions. Conclusion: MaatHRI is the first prototype of UKOSS and other similar platforms in a low and middle income country (LMIC). The model used to develop the platform can be replicated in other LMICs. Funding: Medical Research Council Career Development Award to Manisha Nair (Ref:MR/P022030/1).

\section{Introduction}

Maternal health is a global priority due to the large number of women becoming pregnant every year, an estimated 211 million $^{1}$, and because of the growing disparity in maternal deaths across countries ${ }^{2}{ }^{3}$. India has the highest number of maternal deaths of any country with $\sim 45,000$ deaths yearly ${ }^{2}$. The rate is much higher for some states, such as Assam in the Northeast of India. Assam has nearly half the population of the UK, and 6 women die every day as a result of pregnancy and childbirth complications ${ }^{4}$ compared with around one per week in the $\mathrm{UK}^{5}$. In addition, each year an estimated 5 million pregnant women in India experience a life-threatening complication. To improve care and outcomes, India needs large and robust studies to investigate the risk factors, management and outcomes of pregnancy complications and to find out why disease severity varies from state to state.

In a pilot project (called IndOSS-Assam) we demonstrated the feasibility of setting up a UK-India collaborative platform for maternal and perinatal health research jointly undertaken by Indian clinical collaborators and researchers at the University of Oxford ${ }^{6}$. This was modelled on the UK Obstetric Surveillance System $(\mathrm{UKOSS})^{8}$ and showed that a hospital-based platform can be used to conduct large epidemiological studies and routine surveys to investigate pregnancy complications and management, and establish incidence and outcomes. UKOSS through its work over the past decade has contributed significantly to improving the safety and quality of care for pregnant women. It has inspired several high-income countries to establish obstetric surveillance and research systems which are being used to conduct national and multi-national studies to generate evidence to improve pregnancy care. However, there is no such system in low-and-middle income countries (LMICs) where more than $94 \%$ of all maternal deaths occur.

Our pilot work in India not only justified the importance and urgency, but also demonstrated the need to further adapt and improve the pilot model to create a standardised collaborative platform for both research and research capacity building. This led to the establishment of the Maternal and perinatal Health Research collaboration, India (MaatHRI), a larger standardised collaborative research platform of 14 public and private hospitals across four states in India. The objective of this paper is to describe the processes used to establish and standardise the MaatHRI platform. MaatHRI means mother in Sanskrit.

\section{Methods}

MaatHRI is a hospital-based UK-India collaborative research platform established to: (i) regularly collect data on the prevalence of known and emerging life-threatening pregnancy complications; (ii) conduct large epidemiological studies to generate evidence to improve maternal and perinatal health in India; and (iii) develop research capacity and skills in the collaborating hospitals. It was built on the pilot system, but extensively expanded and standardised over a period of 18 months from May 2017 to September 2018. The following methods were used to establish the collaborative platform:

1. Establishing a network of hospitals and clinical collaborators

2. Setting up a high quality secure system for data collection, storage, and transfer

3. Developing a standardised laboratory infrastructure

4. Implementing regulatory systems

5. Training and supporting collaborators and research staff

Establishing a network of hospitals and clinical collaborators 
Successful completion of the pilot work in two hospitals in Assam allowed us to expand the network from two to nine government hospitals within Assam: six teaching hospitals and four district hospitals. In each hospital, we identified a lead collaborator who were mainly obstetricians. Through their professional networks, we were able to reach out to other hospitals. A hospital was included in the network based on two criteria: (i) willingness of the hospital to participate in a large research collaboration and (ii) a high burden of maternal and perinatal deaths in the population covered by the hospital. Similar to the process used in the pilot work ${ }^{6}$, we mapped the hospitals to assess the spread and coverage of the population in each state.

Setting up a high quality secure system for data collection, storage and transfer

One of the major reasons for success of the pilot work was having dedicated research staff for data collection and data entry. A pragmatic approach was adopted to develop a high quality secure electronic system to overcome the challenges of human resource constraints, lack of dedicated secure computer servers for data storage in the hospitals, and securely sharing data. The following methods were employed:

1. Research nurses were appointed in each hospital and trained

2. Electronic online data collection forms were developed for entering data

3. Data are collated automatically in a cloud-based server located in India

4. Quality assurance and data security procedures were established and implemented

\section{Standardised laboratory infrastructure}

A laboratory infrastructure was created through a partnership with a private laboratory in India, Dr Lal PathLabs (LPL). LPL has a pan-India presence with a network of sample collection centres, regional laboratories and a national reference laboratory in New Delhi, India. Their existing service delivery model was adapted to the requirements of the MaatHRI platform through extensive consultations between the Indian clinical collaborators, and experts at the University of Oxford and LPL. The following services were agreed and are being currently used to standardise the laboratory infrastructure -

Service 1: Provide blood collection kits with instructions to all study hospitals

Service 2: Train MaatHRI research nurses to collect and prepare blood samples

Service 3: Transport samples at ambient conditions from the hospitals to the laboratory

Service 4: Standardise blood assays

Service 5: Produce standardised test reports

We tested the model in a run-in phase before full implementation.

\section{Regulatory systems}

The steering committee constituted for the pilot work (IndOSS-Assam) was expanded to form the MaatHRI steering committee. The committee includes representatives from all the collaborating hospitals, the University of Oxford, Indian policy advocates and experts in statistics and ethics. As MaatHRI is a research platform set up to conduct studies on a long term basis, an independent 'Data safety and monitoring board' (DSMB) was set up including members from India and the UK who are not associated with the MaatHRI platform. A DSMB charter was drafted outlining the roles and responsibilities of the members and how the board will function to provide independent safety review of participants and data, and guidance for observational studies during the course of the ongoing projects. Since the studies currently undertaken through the platform are observational studies, review of adverse event data and reports of serious adverse events (SAEs) are not currently applicable to MaatHRI DSMB. However, should randomised controlled trials be conducted through MaatHRI in the future we would expect the DSMB to be involved in reviewing this type of information.

Funding 
The MaatHRI platform is funded by a Medical Research Council Career Development Award to MN (Ref:MR/P022030/1). The awarded grant was externally peer-reviewed and MN was interviewed by a panel. We are working towards involving the public and patients in the steering committee of the platform. The funder has no role in establishing the platform or writing the paper.

\section{Results}

\section{MaatHRI network of hospitals}

We were able to establish a network of 14 hospitals by September 2018 across four states in India - Assam, Meghalaya, Uttar Pradesh, and Maharashtra. One year after establishing the network, one hospital joined MaatHRI, but two government district hospitals left. A lack of interest in research and high patient load were the main reasons given by the lead collaborators of the departing hospitals. The MaatHRI platform currently includes a network of 14 hospitals (11 Government and 3 private). Figure 1 shows the distribution of the network across India and within the state of Assam. The 14 hospitals together conduct about 100,000 deliveries per year. The network includes an Indian research team of 20 nurses, a project manager, 16 obstetricians, two pathologists, a public health specialist, a general physician and a paediatrician. The UK partners include four clinician epidemiologists from the University of Oxford.

Setting up a high quality and secure system for data collection and storage

Data and biological sample collection: Depending on the patient load and related participant recruitment rates, one or two research nurses have been appointed in each collaborating hospital for the MaatHRI work. The nurses are responsible for recruiting study participants, providing participant information and obtaining informed consent, collecting data and blood samples, and following up participants. The research nurses were specifically trained to undertake these activities. In addition, a project manager has been appointed to manage the research nurses and provide supportive supervision.

Data entry and storage: Our original plan was for research nurses to collect data in online electronic forms using tablet PCs enabling automatic collation in the Microsoft Azure cloud computing platform (Microsoft Corporation) with servers located in India; there is no provision for storing data on the tablets. However, after an initial trial we found that direct data entry in an online form was not possible due to problems with internet connections in several hospitals and the sensitivity associated with a nurse standing with a tablet PC next to a very sick woman. It was therefore decided that paper forms would be used to collect data in these hospitals and the nurse would enter the data immediately afterwards into the online data portal and then destroy the paper forms. Each hospital has a unique Login ID and password to access the data collection forms and their collated data on the online portal.

Quality assurance and data security: The electronic data collection forms have checks and validations to flag logical errors. The project manager is responsible for monitoring data entry on a day-to-day basis. Red flags are raised for errors and incomplete forms immediately so that the research nurse can rectify the errors before the participant is discharged from the hospital. Data stored in the cloud server are encrypted and password protected. Each collaborating hospital can only view and download its own data. Identifiable information are collected for follow-up of participants, but these can only be viewed by the hospital staff and cannot be downloaded by anyone else. Once the data collection is complete, in preparation for analysis, all identifiable information is completely delinked from the clinical data to generate pseudonymised analysis files. We have developed secure mechanisms for transferring data within India and between India and the UK with recommended level of end-end-encryption.

\section{Laboratory infrastructure}

Dr Lal Pathlabs (LPL) provides the laboratory infrastructure for MaatHRI. The following services were tested in a trial run before being fully incorporated into the platform.

Service 1: Blood collection kits with instructions to all study hospitals 
LPL provides the required blood collection kits with specific written guidance to all study hospitals for collecting, processing and packing the blood samples.

Service 2: Train MaatHRI research nurses to collect and prepare blood samples

Technical experts from the laboratory trained the MaatHRI staff (project manager and research nurses) to collect, centrifuge and pack samples before the start of studies. When required, a phlebotomist from their collection centre provided supportive supervision to the research nurses during the initial few weeks to correct or prevent any errors.

The MaatHRI research nurses collect, centrifuge and pack blood samples as per instructions in transportation boxes ready for collection by LPL. A standard test requisition form for each participant is filled in by the obstetrician caring for the participant. This form only includes the participant ID, age and a barcode to ensure participant confidentiality and blinding to minimise reporting bias. The test results are only used for research purposes and not for the provision of clinical care.

Service 3: Transport samples at ambient conditions from the hospitals to the laboratory

A designated person from the LPL collection centre collects the boxes from the hospital. These are transported via road to the nearest regional laboratory where they are checked and then shipped via air to the national laboratory in New Delhi. A flow-chart describing the transportation process from the hospitals to the LPL National Reference Laboratory is shown in Figure 2 and the network is presented in a map in Figure 3. Time in transit is regularly monitored by LPL and reported for each participant along with their test results.

Service 4: Standardising blood assays

All samples are processed and analysed in the LPL National Reference Laboratory based at New Delhi. The assay methods, traceability and performance characteristics are discussed by experts from the University of Oxford's Wolfson laboratory and LPL before including a test in the study. Table 1 shows the traceability and Table 2 shows the performance characteristics for assays that are commonly used for the epidemiological studies undertaken using the MaatHRI platform. The details of specific tests will be presented in subsequent publications. Traceability and assay performance monitoring are important for standardisation of laboratory procedures and quality control. If the quality of a blood sample is compromised in transit, it is not processed and the site-collaborator and research nurse are advised to collect a fresh sample. The laboratory runs quality control checks (QC) daily for each assay (twice a day for some) and monitors their mean coefficient of variation $(\mathrm{CV})$ and standard deviation (SD). The results are shared as part of a performance monitoring plan during monitoring and feedback meetings. In addition, LPL also runs a quarterly Quality Improvement Programme.

The LPL National Reference Laboratory is accredited by the following bodies - College of American Pathologists (CAP); National Accreditation Board for Testing and Calibration (NABL); British Standards Institution (Quality Management System ISO 9001: 2015, FS 60411).

Service - 5: Test reports

Test reports are securely made available to the site-collaborator in each hospital through their usual communication channel. The test report only includes the participant ID, age, hospital name and a laboratory reference number for LPL. Data from the reports are entered in the electronic forms by the research nurse.

\section{Regulatory systems}

MaatHRI steering committee has met biannually since the platform was established in September 2018. The role of the steering committee is to guide the platform in terms of vision, scope, equitable partnership, and research and training priorities. It is also responsible for communicating the results of the studies undertaken through MaatHRI to the Ministry of Health and Family Welfare (MoHFW), Government of India. 
The DSMB periodically reviews participant recruitment, data safety and confidentiality, ethical issues and data quality, and examines whether the overall safety and feasibility of the MaatHRI project is acceptable. The DSMB can be given access to participant data; however, no formal analyses of study outcomes are conducted. Thus, any recommendations to alter study conduct would be based on participant and data safety and not study outcomes. Monitoring of the study will not affect the statistical operating characteristics of the final analysis. The DSMB has met twice since MaatHRI was established in September 2018 and membership includes two Obstetricians (one from the UK and one from India), one Paediatrician (from India), one biostatistician (from the UK), and one expert in bioethics (from India), all with prior experience and expertise in observational epidemiological studies. They were nominated by the study investigators.

\section{Discussion}

\section{Main findings}

MaatHRI, a UK-India collaboration, modelled on UKOSS, was successfully established to conduct hospitalbased research to improve care and outcomes for mothers and babies in India. It includes 14 public and private hospitals across four states in India which together conducts about 100,000 deliveries per year. The platform is standardised in terms of data collection, equipment, and laboratory methodology, and employs strict measures for participant confidentiality and data security. It is monitored by two regulatory bodies, a steering committee and an independent DSMB. Four epidemiological studies have being undertaken through the platform.

\section{Strengths and challenges}

MaatHRI is a collaboration of hospitals that covers deprived populations, some of which are located in remote rural areas of India. While this provides the opportunity to conduct research to improve the health of mothers and babies in areas of the country that have the highest burden of maternal and perinatal deaths, it also poses challenges related to resources and capacity. Appointing new research nurses to collect data and blood samples ensured that the MaatHRI platform was not depriving the hospitals of their scarce human resource. This has created an employment opportunity for nurses in the field of research which is an unusual job for trained nurses. However, the challenge associated with this was the need for extensive training and constant supervision of the nurses. Furthermore, most of the collaborating hospitals had not been involved in a project of this scale and intensity encompassing not just implementation, but designing, standardising and developing the project as equal partners. Therefore, it took more than 20 months of continuous engagement with staff and collaborators to achieve the desired level of quality and standardisation for the MaatHRI platform.

Within the resource constraints, a further challenge is achieving a balance between an ideal collaborative research platform and a pragmatic solution. For example, the ideal platform would have collected data electronically on tablets using online forms, but this was not feasible due to a lack of good internet connectivity in the remote hospitals and cultural sensitivities. Therefore, papers forms are used in some hospitals. However, to mitigate risks and as advised by the DSMB, we have developed a documented process of securely storing and destroying the paper forms within an agreed timeline for each hospital.

Costs related to research staff, standardised laboratory parameters, programming data collection forms, and storing data on Microsoft Azure make studies undertaken through the MaatHRI platform more expensive compared with existing similar systems in the $\mathrm{UK}^{8}$, Europe and Australia ${ }^{9}$. It is our belief, however, that the benefits of generating high quality scientific evidence to answer important and urgent clinical research questions that will save the lives of thousands of future mothers and babies, outweigh these additional costs.

Interpretation (in light of other evidence)

MaatHRI is the first prototype of UKOSS and other similar platforms ${ }^{9}$ in a low and middle income country (LMIC). Within this setting, it covers the most deprived and vulnerable population groups. The MaatHRI platform although built on models of existing surveillance and research platforms in high income countries, is more advanced in terms of using current best practices for standardisation of data and laboratory parameters, 
monitoring data and participant safety, and secure transfer of data within and between countries. All biological samples are analysed at the LPL National Reference Laboratory. The precision, performance and quality of each laboratory parameter are documented and maintained to a high level. The laboratory partnership also benefits from subsidised costs from LPL for each test, at a rate that is $40 \%$ less than their commercial price, with no additional costs for transportation and project management. The laboratory has also started tests for the MaatHRI project which they did not offer previously. This involved completing extensive validation processes. In addition to high quality and standardisation of the laboratory procedures, the pseudonymised laboratory model ensures confidentiality of participants and minimises reporting bias.

Another advantage of the MaatHRI platform is the ability to undertake long term follow-up studies of participants. Identifiable information collected locally from participants helps to locate each participant by hospital staff for follow-up. All studies currently undertaken through the platform have a follow-up component with the potential to generate participant cohorts, based on informed consent, for long term follow-up of the effects of pregnancy complications. Adequate measures have been put in place for securely storing the identifiable information and destroying it after the cohorts for long term follow-up have been established. An independent MaatHRI DSMB monitors data safety and participant confidentiality on an ongoing basis thereby ensuring confidence and trust in the system.

While the platform is established and is currently running three epidemiological studies, the process to develop capacity for research and further improving pregnancy care will continue and is an integral part of the MaatHRI collaboration. The focus is on bi-directional skills development and capacity building through mutual learning between the collaborators in India and the UK. The platform is also being used to develop the research capacity of early career researchers (MSc and PhD students and post-doctoral researchers) interested in working in maternal and perinatal health in an LMIC setting.

\section{Conclusion}

In summary, the methods that we have used to develop the MaatHRI platform make it a unique and high quality research resource using a model that can be replicated in other LMICs. Since being established in September 2018, MaatHRI has already secured further funding, including industry funding. One epidemiological study is complete and three others are in various stages of participant recruitment and data collection. In addition to the DSMB and steering committee, we are working to achieve a more active and extensive process to involve the public and patients (pregnant women, mothers and their families) and civil societies working to improve the health and wellbeing of mothers and babies in India. We also intend to make the data generated through the MaatHRI platform available to researchers for secondary analysis, and are developing a data sharing governance policy for MaatHRI. In addition to research impact, our approach to building the platform on the premise of equitable partnership between all collaborators and developing research capacity in the collaborating institutions will further contribute to the sustainability of MaatHRI.

\section{Acknowledgments}

We thank Prof. U C Sarma, retired Vice Chancellor of Srimanta Sankaradeva University of Health Sciences, Guwahati, Assam for his valuable contribution in establishing the MaatHRI platform. We also thank Prof. Hem Kanta Sarma, Professor and Head of the Department of Obstetrics and Gynaecology, Jorhat Medical College and Hospital, Assam for his contribution during the initial phase of setting up MaatHRI.

\section{Competing interests}

The authors declare that they have no competing interests.

\section{Authorship and contributions}

MN developed the concept for the MaatHRI platform, led the work as the chief investigator to establish MaatHRI, and wrote the first draft of the paper. BB, AB, KB, SC, SCC, AC, DD, GD, VAI, SDK, RMK, PK, PM, RM, PN, MCR, AR, IR, US, CSV, RKT, and FZ contributed equally, and their names are included in the alphabetic order of their last name. They are collaborators and investigators of the MaatHRI project 
and led the work in their respective institution to establish the platform. They also edited the paper. MH, NK and RN contributed to developing the laboratory infrastructure for MaatHRI and edited the paper. CB, MK, JJK are advisors and have contributed to developing the MaatHRI platform and editing the paper.

\section{Ethics statement}

The MaatHRI platform and the ongoing studies have been approved by the institutional review boards (IRB) of each coordinating Indian institution, namely: Srimanta Sankaradeva University of Health Sciences, Guwahati, Assam (No.MC/190/2007/Pt-1/126); Nazareth hospital, Shillong, Meghalaya (Ref No. NH/CMO/IEC/COMMUNICATIONS/18-01); Emmanuel Hospital Association, New Delhi (Ref. Protocol No.167); Mahatma Gandhi Institute of Medical Sciences, Sevagram, Maharashtra (Ref No. MGIMS/IEC/OBGY/118/2017); and the Institute of Medical Sciences, Banaras Hindu University, Varanasi, Uttar Pradesh (No.Dean/2018/EC/290). The project has also been approved by the Government of India's Health Ministry's Screening Committee, the Indian Council of Medical Research, New Delhi (ID number 2018-0152) and by the Oxford Tropical Research Ethics Committee (OxTREC), University of Oxford, UK (OxTREC Ref: 7-18).

\section{Funding statement}

The MaatHRI platform is funded by a Medical Research Council Career Development Award to MN (Ref:MR/P022030/1). The funder has no role in establishing the platform, study design, or writing the paper.

\section{References}

1. World Health Organization. The World health report : 2005 : make every mother and child count. Geneva: World Health Organization, 2005.

2. Graham W, Woodd S, Byass P, et al. Diversity and divergence: the dynamic burden of poor maternal health. The Lancet 2016 doi: 10.1016/S0140-6736(16)31533-1

3. WHO, UNICEF, UNFPA, et al. Maternal mortality: Levels and trends 2000 to 2017. Geneva: World Health Organisation, 2019.

4. Office of Registrar General and Census Commissioner India. Annual Health Survey 2012-13: Fact sheet. New Delhi: Ministry of Home Affairs, Government of India, 2014.

5. Knight M, Bunch K, Tuffnell D, et al. Saving Lives, Improving Mothers' Care - Lessons learned to inform maternity care from the UK and Ireland Confidential Enquiries into Maternal Deaths and Morbidity 2015-17. Oxford: National Perinatal Epidemiology Unit, University of Oxford, 2019.

6. Nair M, Choudhury MK, Choudhury SS, et al. IndOSS-Assam: Investigating the feasibility of introducing a simple maternal morbidity surveillance and research system in Assam, India. BMJ global health 2016;1(1):e000024.

7. Nair M, Choudhury MK, Choudhury SS, et al. The association between maternal anaemia and pregnancy outcomes: a cohort study in Assam, India.BMJ Global Health 2016;1:e000026. doi: 10.1136/bmjgh-2015000026

8. Knight M, Lindquist A. The UK Obstetric Surveillance System: Impact on patient safety. Best Practice \& Research Clinical Obstetrics \& Gynaecology 2013;27(4):621-30. doi: https://doi.org/10.1016/j.bpobgyn.2013.03.002

9. Knight M. The International Network of Obstetric Survey Systems (INOSS): benefits of multi-country studies of severe and uncommon maternal morbidities. Acta obstetricia et gynecologica Scandinavica 2014;93(2):127-31. doi: 10.1111/aogs.12316 [published Online First: 2014/01/03]

Figure - 1: Distribution of the MaatHRI collaborating hospitals and regions covered by the hospitals across India 
Figure - 2: A flow-chart showing the transportation of samples from the hospital to the Dr Lal PathLabs National Reference Laboratory for processing and analysis

Figure - 3: Laboratory network for the MaatHRI platform

Table-1: Traceability of Assays

Table-2: Assay Information and performance characteristic.

Table-1: Traceability of Assays

\begin{tabular}{lll}
\hline Sl No & Name of test & Calibrator traceability (reference material/ reference method) \\
\hline 1 & Haemoglobin & 1:250 dilution in NCCLS2 recommended reagent for the hemiglobincyanide cyanmet \\
2 & Hematocrit & Calculated \\
3 & Platelets & A 1:101 dilution is made using a $20 \mu \mathrm{L}$ TC pipette and $2 \mathrm{~mL}$ of $1 \%$ filtered ammoni \\
4 & Serum Ferritin & WHO 3rd International Standard $94 / 572$ \\
5 & Haemoglobin electrophoresis & NGSP Certification for A2/F \\
\hline
\end{tabular}

NGSP - National Glycohemoglobin Standardization Program; CLSI - Clinical and Laboratory Standards Institute; HbF - Fetal haemoglobin; HbA2 - Haemoglobin Subunit Alpha 2; NA - Not applicable

Table-2: Assay Information and performance characteristics

\begin{tabular}{lll}
\hline Name of test & System used for the analysis & Method information (supplier/method) \\
\hline Haemoglobin & DxH -800 (Beckman coulter) & Photometric \\
Hematocrit & DxH -800 (Beckman coulter) & Automated calculation \\
Platelets & DxH -800 (Beckman coulter) & Impedance/coulter principle \\
Serum Ferritin & Siemens ADVIA Centaur & Chemiluminescence Immunoassay (CLI \\
Haemoglobin electrophoresis & Variant II Hemoglobin testing system (BIO-RAD) & High Performance Liquid Chromatogra] \\
\hline
\end{tabular}

CAP - College of American Pathologists; CAP PT - College of American Pathologists Proficiency Testing programme; HbF - Fetal haemoglobin; HbA2 - Haemoglobin Subunit Alpha 


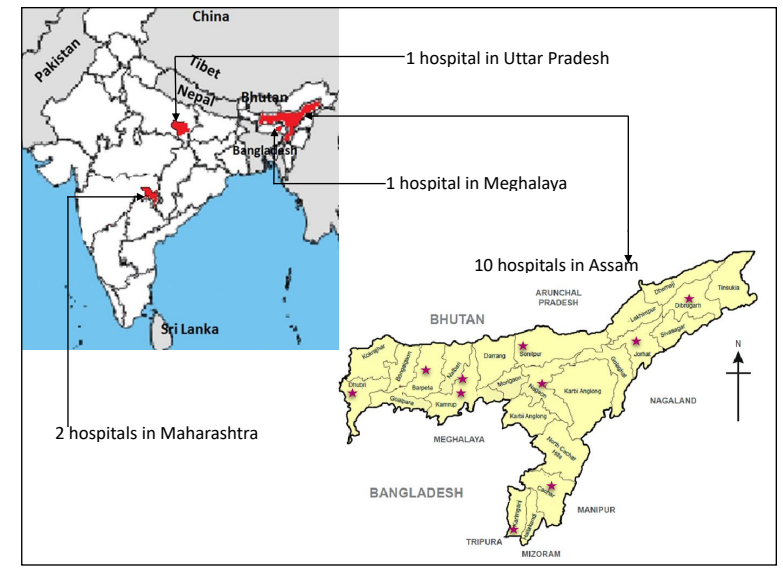

Figure - 1: Distribution of the MaatHRI collaborating hospitals and regions covered by the hospitals across India 


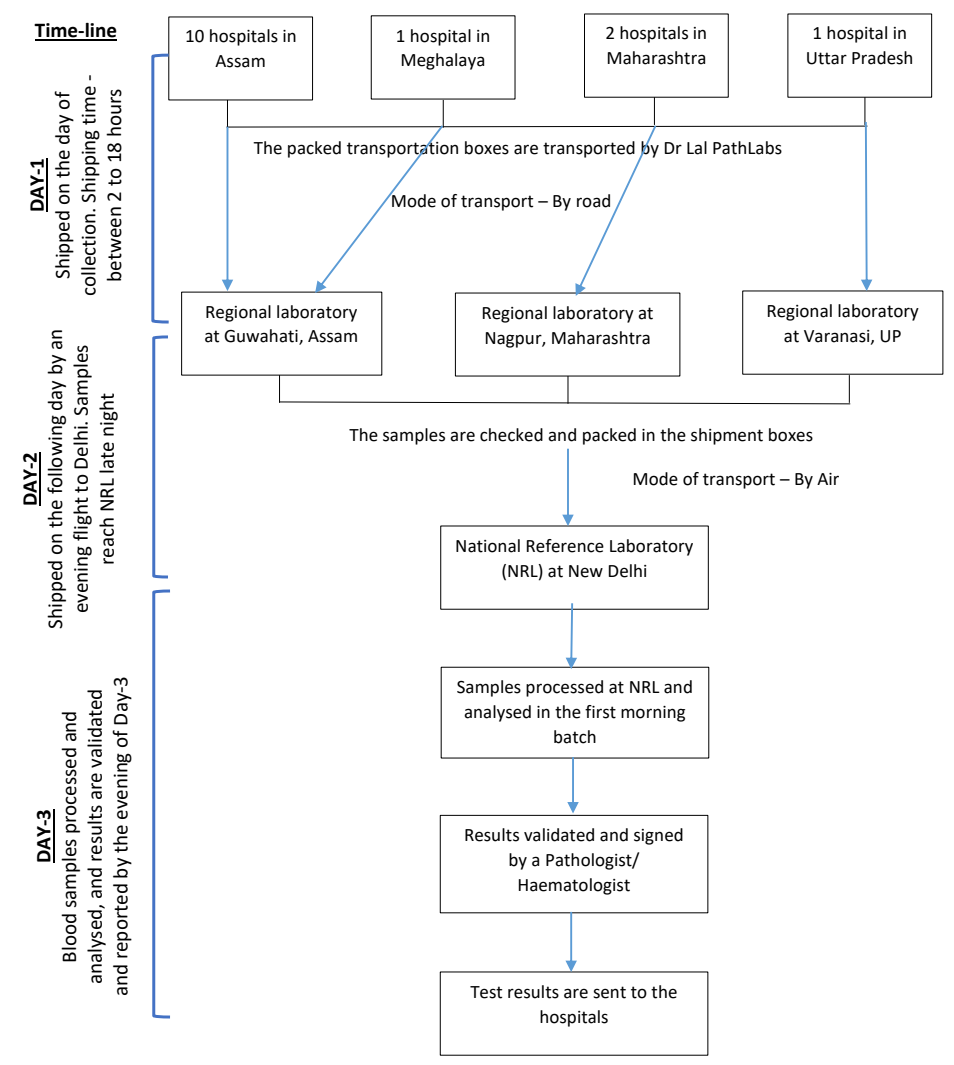

Figure - 2: A flow-chart showing the transportation of samples from the hospital to the Dr Lal PathLabs National Reference Laboratory for processing and analysis 


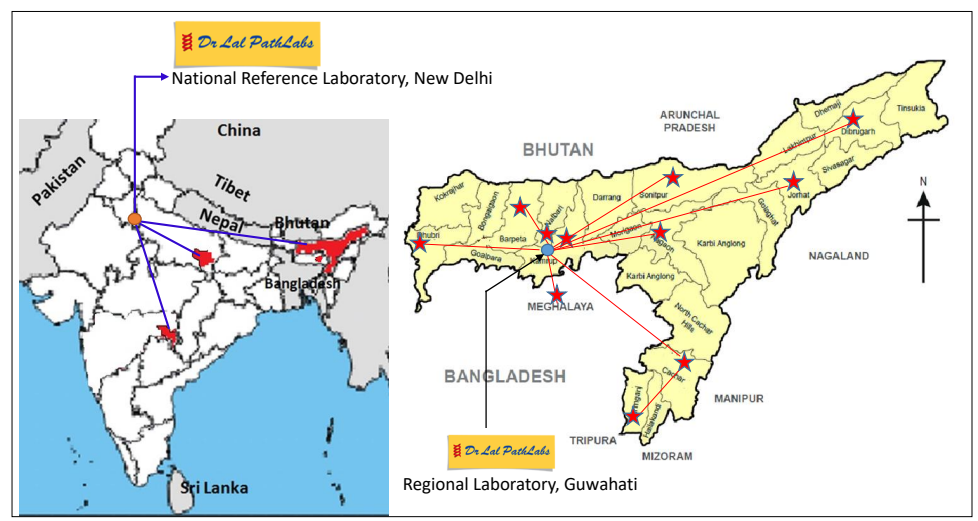

Figure - 3: Laboratory network for the MaatHRI platform 